Article

\title{
Uridine Diphosphate-Dependent Glycosyltransferases from Bacillus subtilis ATCC 6633 Catalyze the 15-O-Glycosylation of Ganoderic Acid A
}

\author{
Te-Sheng Chang ${ }^{1,+}+\mathbb{D}$, Jiumn-Yih $\mathrm{Wu}^{2,+}$, Tzi-Yuan Wang ${ }^{3} \mathbb{C}$, Kun-Yuan $\mathrm{Wu}^{1}$ \\ and Chien-Min Chiang $4, *$ (D) \\ 1 Department of Biological Sciences and Technology, National University of Tainan, Tainan 70005, Taiwan; \\ mozyme2001@gmail.com (T.-S.C.); joy0067tw@gmail.com (K.-Y.W.) \\ 2 Department of Food Science, National Quemoy University, Kinmen County 892, Taiwan; wujy@nqu.edu.tw \\ 3 Biodiversity Research Center, Academia Sinica, Taipei 115, Taiwan; tziyuan@gmail.com \\ 4 Department of Biotechnology, Chia Nan University of Pharmacy and Science, No. 60, Sec. 1, Erh-Jen Rd., \\ Jen-Te District, Tainan 71710, Taiwan \\ * Correspondence: cmchiang@mail.cnu.edu.tw; Tel.: +886-82-313-310; Fax: +88-6-82-313-797 \\ + These authors contributed equally to this work.
}

Received: 11 October 2018; Accepted: 3 November 2018; Published: 5 November 2018

\begin{abstract}
Bacillus subtilis ATCC (American type culture collection) 6633 was found to biotransform ganoderic acid A (GAA), which is a major lanostane triterpenoid from the medicinal fungus Ganoderma lucidum. Five glycosyltransferase family 1 (GT1) genes of this bacterium, including two uridine diphosphate-dependent glycosyltransferase (UGT) genes, BsUGT398 and BsUGT489, were cloned and overexpressed in Escherichia coli. Ultra-performance liquid chromatography confirmed the two purified UGT proteins biotransform ganoderic acid A into a metabolite, while the other three purified GT1 proteins cannot biotransform GAA. The optimal enzyme activities of BsUGT398 and BsUGT489 were at $\mathrm{pH} 8.0$ with $10 \mathrm{mM}$ of magnesium or calcium ion. In addition, no candidates showed biotransformation activity toward antcin $\mathrm{K}$, which is a major ergostane triterpenoid from the fruiting bodies of Antrodia cinnamomea. One biotransformed metabolite from each BsUGT enzyme was then isolated with preparative high-performance liquid chromatography. The isolated metabolite from each BsUGT was identified as ganoderic acid A-15-O- $\beta$-glucoside by mass and nuclear magnetic resonance spectroscopy. The two BsUGTs in the present study are the first identified enzymes that catalyze the 15-O-glycosylation of triterpenoids.
\end{abstract}

Keywords: ganoderic acid; Bacillus subtilis; biotransformation; UDP-glycosyltransferase

\section{Introduction}

The fundamental step in developing new drugs is finding new compounds. In addition to synthetic and natural sources, biotransformation is an alternative route for obtaining new compounds. Xenobiotics can be biotransformed into new compounds via microorganisms or enzymes. Based on the numerous bioactivities of triterpenoids, several scientists have focused on studying triterpenoid biotransformations to find new bioactive triterpenoids [1-4].

Ganoderma lucidum (the Chinese name for which is "Lingzhi") has numerous bioactive constituents, such as polysaccharides and triterpenoids [5]; more than 300 different triterpenoids have been identified from Ganoderma spp. [6]. Ganoderic acid A (GAA) is a typical Ganoderma triterpenoid [7] which has been validated with many bioactivities [8-13]. Although hundreds of bioactive triterpenoids have been 
isolated from G. lucidum, few studies have focused on the biotransformation of Ganoderma triterpenoids. On the other hand, our previous study showed that Bacillus subtilis ATCC (American type culture collection) 6633 biotransforms antcin K, a major ergostane triterpenoid from the fruiting bodies of Antrodia cinnamomea, to its glycoside derivatives [14]. In the present study, the B. subtilis strain was confirmed to biotransform the most abundant Ganoderma triterpenoid GAA as well. Therefore, we were interested in examining the key enzymes of B. subtilis ATCC 6633 in the biotransformation of GAA and antcin $\mathrm{K}$ triterpenoids.

In nature, glycosylation is catalyzed by glycosyltransferases (GTs, EC 2.4.x.y), which transfer sugar moieties from the activated donor molecules to specific acceptor molecules [15-17]. According to the Carbohydrate-Active Enzymes (CAZy) database, GTs are now classified into 105 families [18]. Among them, some glycosyltransferases family 1 (GT1) proteins were found to use small molecules, such as flavonoids or terpenes, as sugar acceptors [15-17]. Furthermore, uridine diphosphate (UDP)-dependent glycosyltransferases (UGTs), which belong to the GT1 gene family, can use UDP-glucose as the sugar donor $[15,18]$. Thus, five GT1 genes, including two UGT genes, were cloned from the B. subtilis ATCC 6633 strain and overexpressed in Escherichia coli. The biotransformation activities of the expressed enzymes were confirmed and optimized in this study. The metabolite biotransformed by the functional enzymes was then isolated and identified.

\section{Results}

\subsection{Confirming Biotransformation of GAA by Bacillus Subtilis ATCC 6633}

To confirm whether B. subtilis ATCC 6633 could biotransform GAA, the bacterium was cultivated in broth with GAA, and the fermentation broth was analyzed using ultra-performance liquid chromatography (UPLC).

Figure 1 shows the UPLC of the initial (dashed line) and $24 \mathrm{~h}$ (solid line) fermentation broths of the strain B. subtilis ATCC 6633 with GAA. The precursor GAA appeared at a retention time (RT) of 8.6 min. After $24 \mathrm{~h}$ fermentation, the peak of the precursor decreased, while two new peaks-a major peak, compound (1), with a RT of $7.0 \mathrm{~min}$ and a minor peak, compound (2) with a RT of $6.3 \mathrm{~min}$-appeared. Moreover, the two new peaks did not appear at $24 \mathrm{~h}$ of the fermentation broths of the strain in the absence of GAA (Figure S1). The results confirmed that GAA was biotransformed by the strain B. subtilis ATCC 6633.

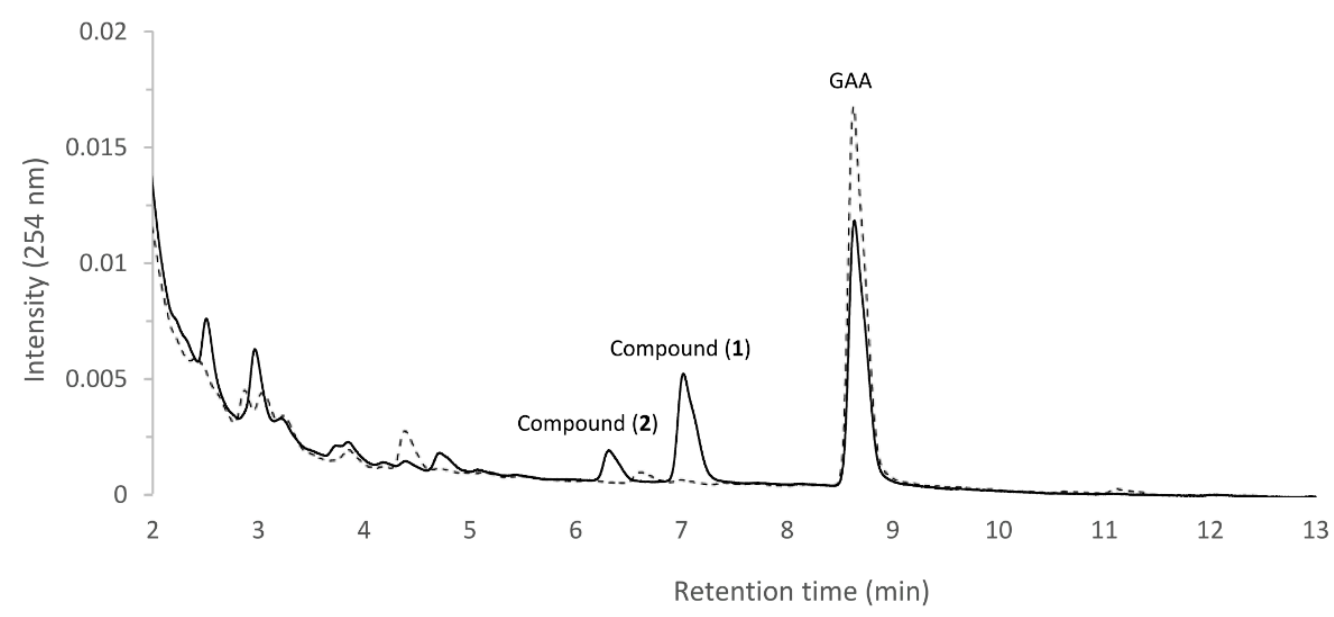

Figure 1. Biotransformation of ganoderic acid A (GAA) by B. subtilis ATCC (American type culture collection) 6633. The strain was cultivated in modified glucose-nutrient (MGN) media containing $0.02 \mathrm{mg} / \mathrm{mL}$ of ganoderic acid A (GAA). The initial cultivation (dashed line) and the $72 \mathrm{~h}$ cultivation (solid line) of the fermentation broth were analyzed with ultra-performance liquid chromatography (UPLC). The UPLC operation conditions are described in the Materials and Methods section. 


\subsection{Phylogenetic Analysis of GTs from B. subtilis ATCC 6633}

Previous studies showed three microbial UGTs were validated with triterpenoid glycosylation activity, including BsYjiC (GenBank Protein Accession No. NP_389104) from B. subtilis 168 [19,20], UGT109A1 (GenBank Protein Accession No. ASY97769) from B. subtilis CTCG 63501 [21], and BsGT1 (GenBank Protein Accession No. ANP92054) from B. subtilis KCTC 1022 [22]. To classify which GT genes of B. subtilis ATCC 6633 are responsible for the biotransformation of GAA, a phylogenetic analysis of 30 annotated GTs from the B. subtilis ATCC 6633 genome (GenBank BioProject Accession No. PRJNA43011) was compared with the three validated microbial UGTs (Figure 2).

Among the 30 GTs, nine GT1 family genes (star in Figure 2), including two UGTs (BsUGT398 and BsUGT489), were identified as putative gene candidates. The results revealed BsUGT489 (GenBank Protein Accession No. WP_003220489) of B. subtilis ATCC 6633 was clustered with three validated UGTs (gray background in Figure 2). BsUGT398 (GenBank Protein Accession No. WP_003225398), BsGT110 (GenBank Protein Accession No. WP_003220110), BsGT292 (GenBank Protein Accession No. WP_032727292), and BsGT296 (GenBank Protein Accession No. WP_003219296) were closely related to the UGT cluster. Thus, the five candidates (bold text in Figure 2) were selected for further functional assay.

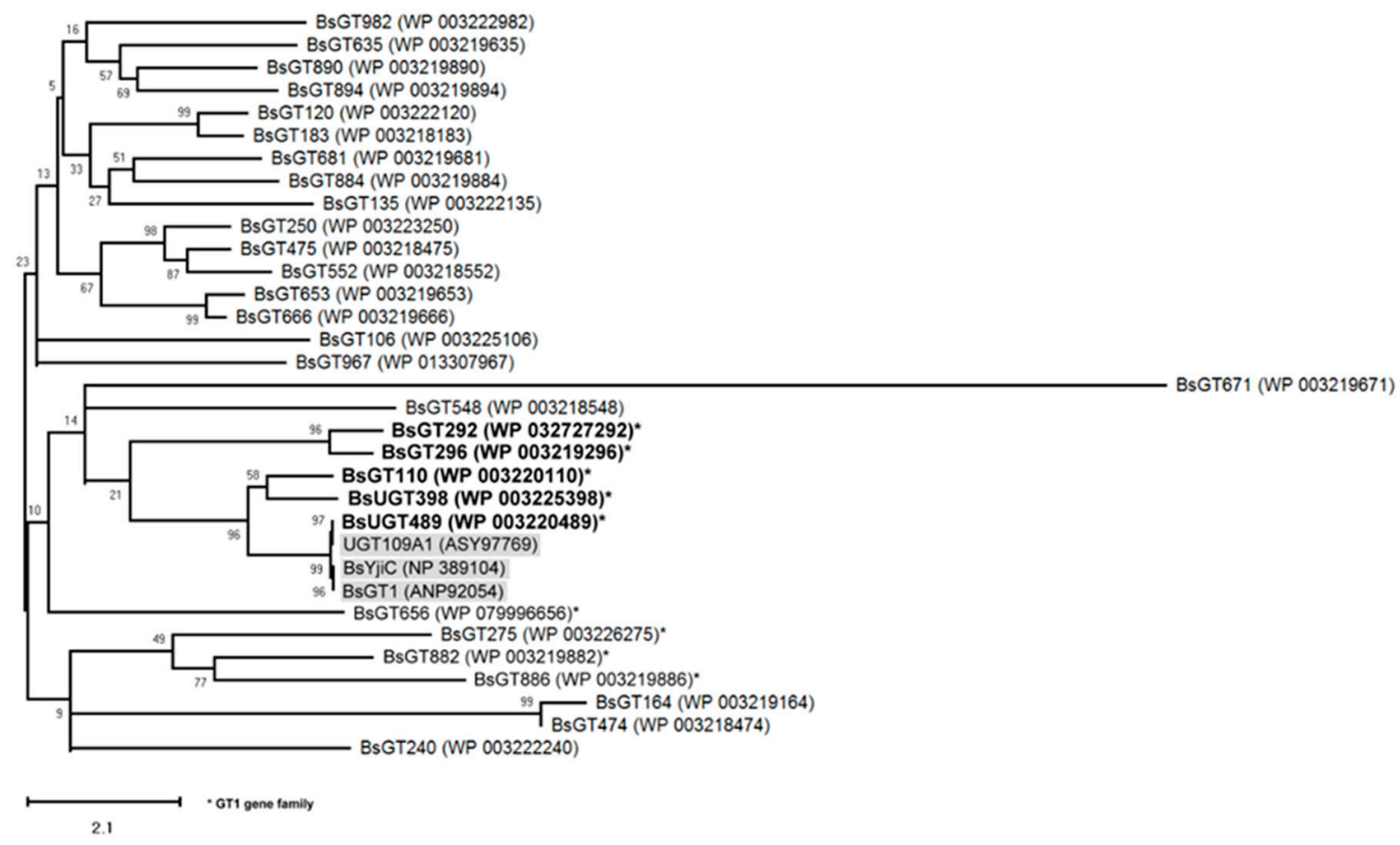

Figure 2. Molecular phylogenetic analysis of BsGT candidates inferred with the maximum likelihood (ML) method. The best-fit ML model selection is mtREV24 [23]. The tree with the highest log likelihood $(-6498.11)$ is shown. The percentage of trees in which the associated taxa clustered together is shown next to the branches. Initial tree(s) for the heuristic search were obtained automatically by applying the neighbor-join and BioNJ algorithms to a matrix of pairwise distances estimated using a JTT model and then selecting the topology with the superior log likelihood value. The tree is drawn to scale, with the branch lengths measured in the number of substitutions per site. The analysis involved 10 amino acid sequences. All positions with less than 95\% site coverage were eliminated. That is, fewer than $5 \%$ alignment gaps, missing data, and ambiguous bases were allowed at any position. There were a total of 372 positions in the final dataset. Evolutionary analyses were conducted in MEGA X [24]. 


\subsection{Cloning and Overexpression of GT1 from B. subtilis ATCC 6633 in E. coli}

Among the five GT1 genes of B. subtilis ATCC 6633, three GT1 genes (BsGT110, BsGT292, BsGT296) had been previously subcloned into the pETDuet-1 ${ }^{\mathrm{TM}}$ expression vector, overexpressed in E. coli BL-21 (DE3), and the produced GT1 proteins were purified with $\mathrm{Ni}^{2+}$ chelate affinity chromatography [25]. Thus, the three purified GT1 enzymes were ready for use in this study. The other two GT1 genes (BsUGT398, and BsUGT489) were newly amplified from B. subtilis ATCC 6633 genomic DNA with polymerase chain reaction (PCR) with specific primer sets (Table S1) and subcloned into the pETDuet-1 expression vector to form pETDuet-BsUGT (Figure S2a). The recombinant BsUGT was overexpressed in E. coli. The produced protein was purified and analyzed with sodium dodecyl sulfate polyacrylamide gel electrophoresis (SDS-PAGE) (Figure S2b). Thus, the five purified GT1 proteins were used for another biotransformation assay.

\subsection{Activity Assays of GT1 from B. subtilis ATCC 6633 Toward GAA}

The five purified GT1 proteins were incubated with UDP-glucose and the precursor GAA. After a 30 min reaction, the reaction mixtures were analyzed with UPLC. Only BsUGT398 and BsUGT489 had biotransformation activity toward GAA, while the other three GT1 proteins of B. subtilis ATCC 6633 did not have this activity. Because our previous study showed that BsGT110 exhibited glycosylation activity toward 8-hydroxydaidzein [25], the reason for the lack of activity of the GT1 toward GAA is the substrate specificity. However, neither BsGT292 nor BsGT296 were active toward GAA and 8-hydroxydaidzein. The substrates of the two BsGTs are not known yet. The UPLC results further revealed the biotransformed metabolite in either the BsUGT398 (Figure 3a) or BsUGT489 (Figure 3b) reaction had an identical RT of $7.0 \mathrm{~min}$, which was the same as the RT of compound (1) in the GAA biotransformation by the strain B. subtilis ATCC 6633 (Figure 1). Thus, this result implied that the BsUGT enzymes and the strain B. subtilis ATCC 6633 might have the same or similar biotransformation activity toward GAA.

In a previous study, we showed that B. subtilis ATCC 6633 could glycosylate antcin K, a major ergostane triterpenoid from the fruiting bodies of Antrodia cinnamomea [14]. Thus, we speculated that the five GT1 enzymes might have the ability to biotransform antcin K. The purified GT1 proteins were also incubated with UDP-glucose and the precursor antcin $\mathrm{K}$. After a $30 \mathrm{~min}$ reaction, the reaction mixtures were analyzed with UPLC. Unfortunately, the results showed that no metabolite was biotransformed by the five GT1 proteins.

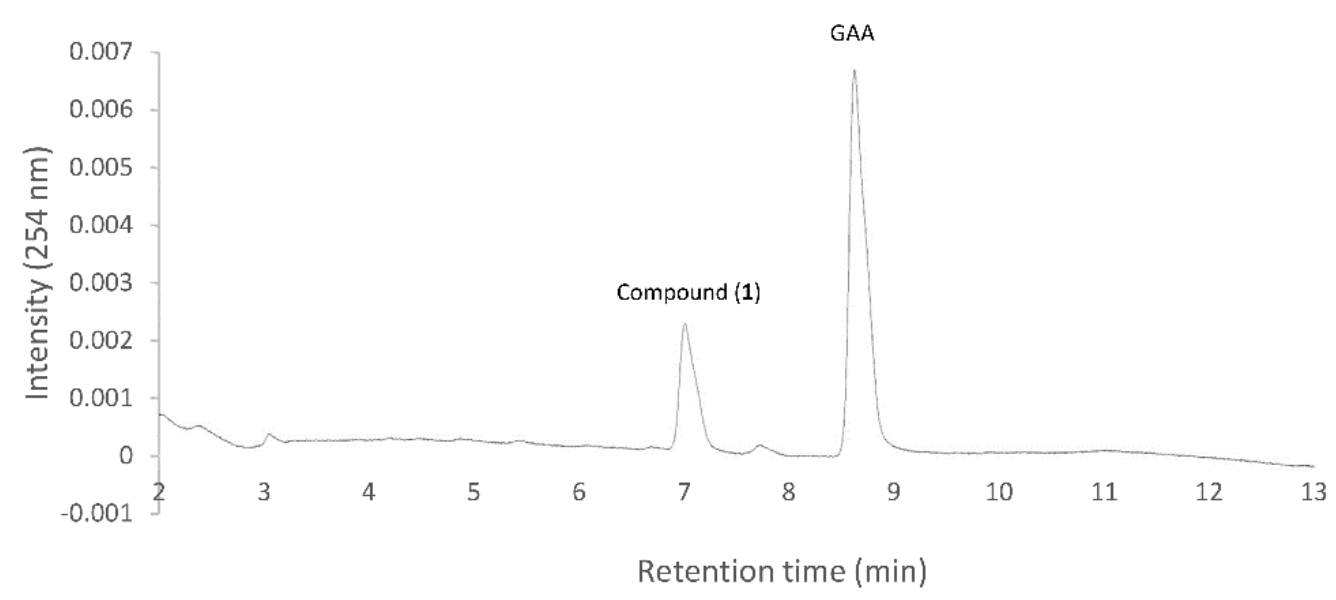

(a)

Figure 3. Cont. 


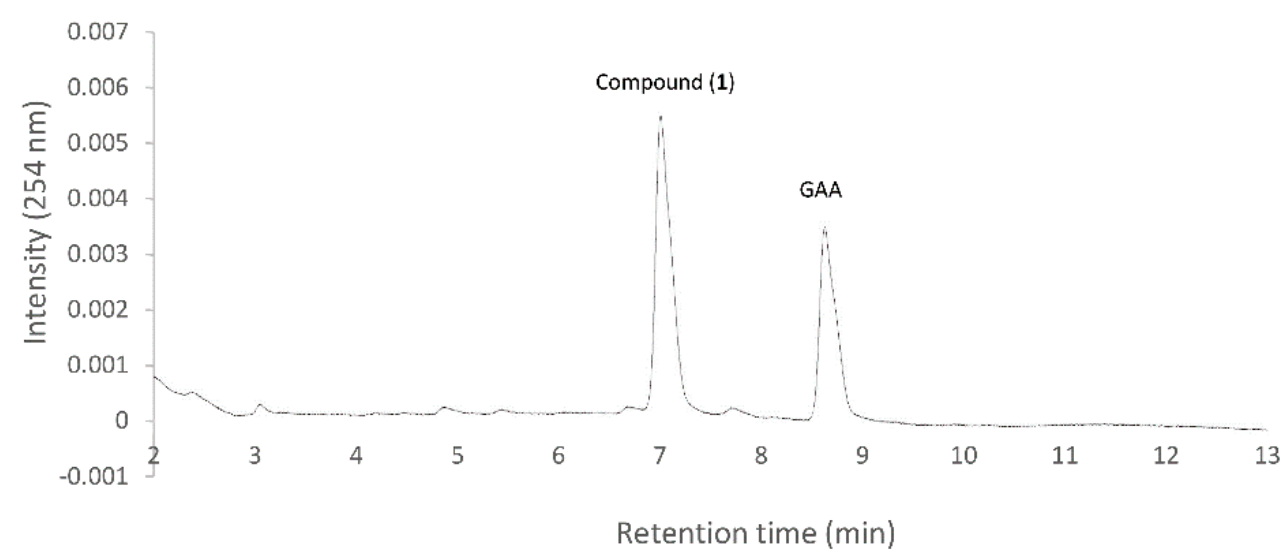

(b)

Figure 3. Biotransformation of GAA by purified BsUGT398 (a) or BsUGT489 (b). Two micrograms of purified BsUGT398 or BsUGT489 were incubated with $0.4 \mathrm{mM}$ uridine diphosphate (UDP)-glucose and $0.02 \mathrm{mg} / \mathrm{mL}$ of GAA in the presence of $50 \mathrm{mM}$ Tris $\mathrm{pH} 8.0$ and $10 \mathrm{mM}$ of $\mathrm{MgCl}_{2}$. After $30 \mathrm{~min}$ incubation, the mixtures were analyzed with UPLC. The UPLC conditions are described in Materials and Methods.

\subsection{Optimal Catalyzing Conditions for BsUGT398 and BsUGT489}

The activities of purified BsUGT398 and BsUGT489 were determined at different $\mathrm{pH}$ values, temperatures, and metal ions. Many GTs utilize divalent metal ion cofactors, and $\mathrm{Mg}^{2+}$ was conjugated in the native crystals of some GTs [26]. In addition, $\mathrm{Ca}^{2+}$ and $\mathrm{Mn}^{2+}$ were also used in the crystal structure studies of GTs [27]. Therefore, we decided to use the three metal ions to optimize the activities of the two BsUGTs. On the other hand, to expand the testing range of the metal ions, a potentially inhibitory cation, $\mathrm{Cd}^{2+}$, was also used. The result is shown in Figure 4. The optimal catalyzing condition for each UGT protein is $\mathrm{pH} 8.0$ with $10 \mathrm{mM}$ of $\mathrm{Mg}^{2+} \mathrm{or} \mathrm{Ca}^{2+}$. The activity of the two UGTs decreased by about $70 \%$ in the absence of either the $\mathrm{Mg}^{2+}$ or $\mathrm{Ca}^{2+}$ metal ion and was completely inhibited in the presence of $\mathrm{Cd}^{2+}$. For temperature, BsUGT398 and BsUGT489 had optimal activity at 40 and $30{ }^{\circ} \mathrm{C}$, respectively.

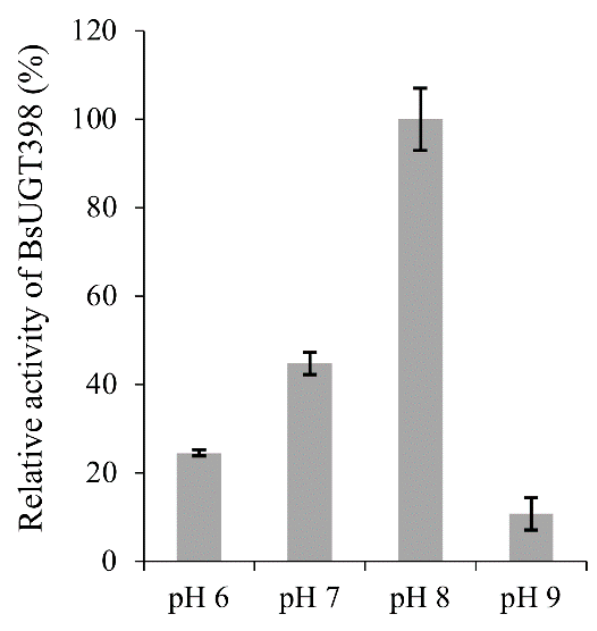

(a)

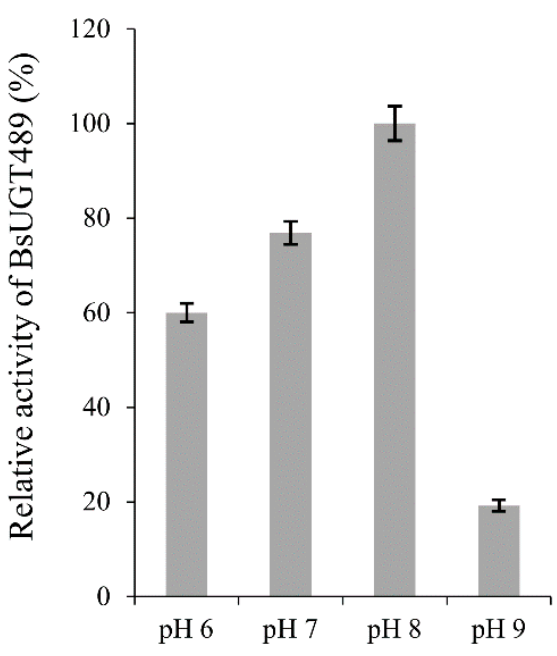

(b)

Figure 4. Cont. 


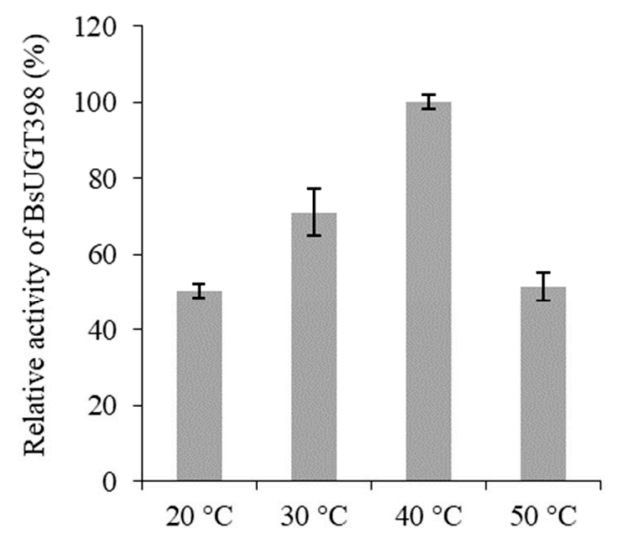

(c)

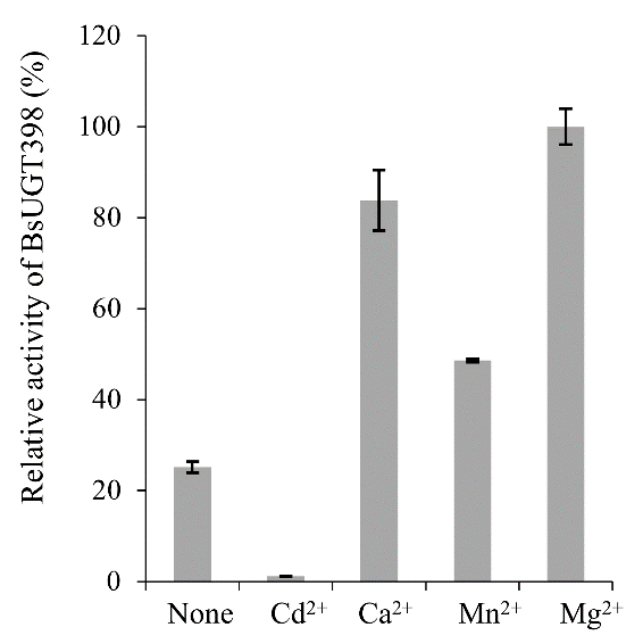

(e)

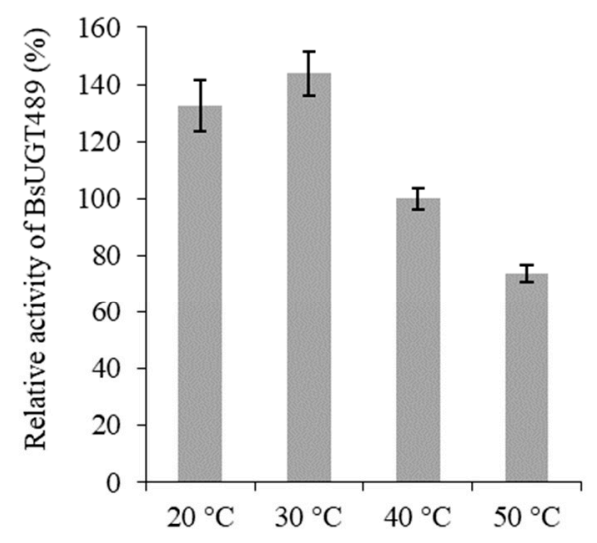

(d)

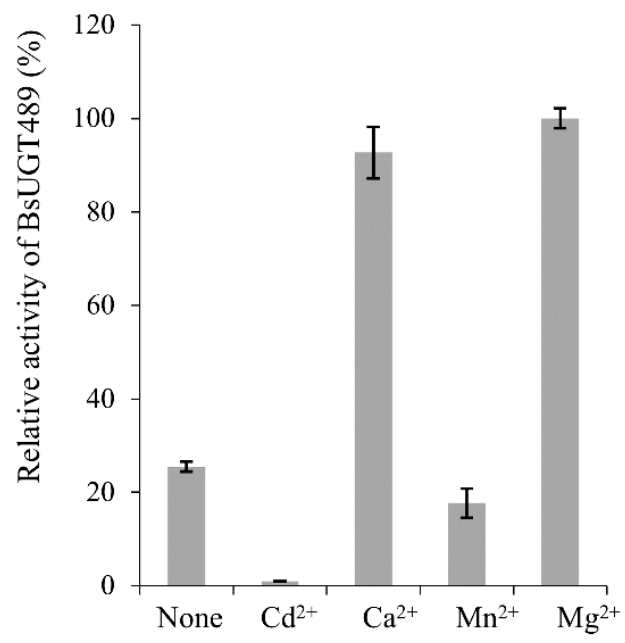

(f)

Figure 4. Effects of $\mathrm{pH}(\mathbf{a}, \mathbf{b})$, temperature (c,d), and metal ion (e,f) on BsUGT398 (a,c,e) or BsUGT489 $(\mathbf{b}, \mathbf{d}, \mathbf{f})$ activity. The standard condition was set as $2 \mu \mathrm{g}$ of the purified enzyme, $1 \mathrm{mg} / \mathrm{mL}$ of GAA, $10 \mathrm{mM}$ of $\mathrm{MgCl}_{2}$, and $10 \mathrm{mM}$ of uridine diphosphate (UDP)-glucose in $50 \mathrm{mM}$ of Tris at $\mathrm{pH} 8.0$ and $40{ }^{\circ} \mathrm{C}$. To determine the optimal reaction condition, the $\mathrm{pH}$, temperature, or metal ion in the standard condition was replaced with the tested condition. Relative activity was obtained by dividing the area of the summation of the product peak of the reaction in the UPLC profile with that of the reaction at the standard condition. The mean $(n=3)$ is shown, and the standard deviations are represented by error bars.

\subsection{Isolation and Identification of the Biotransformation Metabolite}

To resolve the chemical structures of the metabolites, the biotransformation was scaled up by either BsUGT398 or BsUGT489, and the metabolite from each biotransformation was purified with preparative high-performance liquid chromatography (HPLC). From a $40 \mathrm{~mL}$ reaction mixture containing $20 \mu \mathrm{g} / \mathrm{mL}$ of BsUGT, $1 \mathrm{mg} / \mathrm{mL}$ of GAA, $10 \mathrm{mM}$ of UDP-glucose, $10 \mathrm{mM}$ of $\mathrm{MgCl}_{2}$, and $50 \mathrm{mM}$ of Tris $\mathrm{pH} 8.0,15.1 \mathrm{mg}$ and $37.4 \mathrm{mg}$ of the metabolite, compound (1), were isolated from the biotransformation by BsUGT398 and BsUGT489, respectively. The metabolite from each biotransformation was elucidated with electrospray ionization mass spectrometry (ESI-MS) and nuclear magnetic resonance (NMR) spectra. They showed identical mass and ${ }^{13} \mathrm{C}-\mathrm{NMR}$ data. The mass data showed an $[\mathrm{M}-\mathrm{H}]^{-}$ion peak at $m / z: 677.67$ in the ESI-MS spectrum, corresponding to $\mathrm{C}_{36} \mathrm{H}_{53} \mathrm{O}_{12}$ that indicated the glucosylation of GAA. The structure of the metabolites was further confirmed 
as GAA-15-O- $\beta$-glucoside using NMR data, by comparison with that in our previously reported study [28]. The NMR spectrum of the two metabolites from either BsUGT398 or BsUGT489 are shown in Figure S3-S16.The biotransformation process of GAA by either BsUGT398 or BsUGT489, where D-glucose was tentatively assumed, is shown in Figure 5.

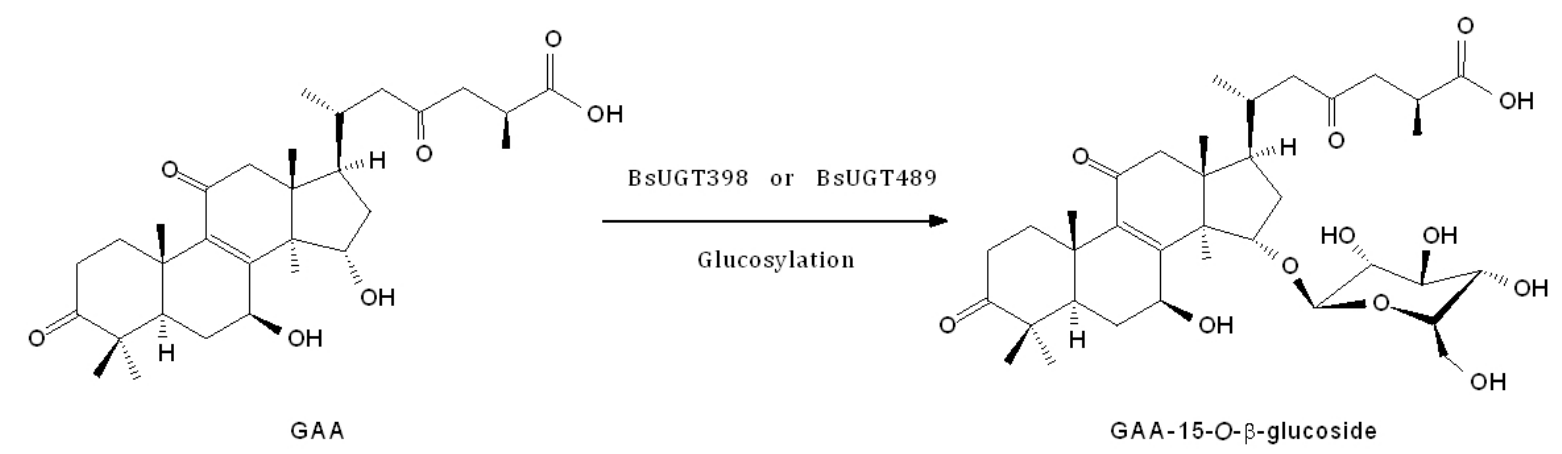

Figure 5. Biotransformation process of GAA by BsUGT398 or BsUGT489.

\section{Discussion}

Before the present study, three microbial UGTs—BsYjiC [19,20], UGT109A1 [21], and BsGT1 [22]— had been demonstrated to possess triterpenoid glycosylation activity. These UGTs catalyze the C-3, C-6, C-12, or C-20 carbon positions with a hydroxyl group for glycosylation of triterpenoids. Accordingly, BsUGT398 and BsUGT489 from B. subtilis ATCC 6633 that catalyzes the C-15 glycosylation of GAA are unique, and the glycosylation site on the triterpenoids is also new. Only one metabolite, glycosylation on the C-15 hydroxyl group, was biotransformed by the two BsUGTs, in spite of the other C-7 hydroxyl or C-26 carboxyl groups of GAA available for glycosylation (Figure 5). Moreover, neither of the two BsUGTs catalyzed the glycosylation of antcin K, which contains C-3, C-4, and C-7 hydroxyl and C-26 carboxyl groups but lacks the C-15 hydroxyl group available for glycosylation in the structure. The results imply that the two BsUGTs have regio- and substrate selection of glycosylation of triterpenoids.

B. subtilis ATCC 6633 has been reported to possess glycosylation activity toward the pentacyclic triterpenoids oleanolic acid, echinocystic acid, or betulinic acid [29] or the C-26 carboxyl group of tetracyclic ergostane triterpenoid, antcin K [14]. Moreover, all the glycosylation activity by the strain were at carboxyl groups of the triterpenoid precursors. However, five GT1 enzymes of $B$. subtilis ATCC 6633 lacked biotransformation activity toward antcin K. Therefore, other enzymes must be responsible for catalyzing glycosylation on the carboxyl groups of the triterpenoids in the B. subtilis ATCC 6633. Nevertheless, previously identified triterpenoid-glycosylation UGT enzymes, including BsYjiC [19,20], UGT109A1 [21], BsGT1 [22], BsUGT398, and BsUGT489 (the present study), have been proven to catalyze the glycosylation of triterpenoids only on the hydroxyl groups of triterpenoids. Thus, the other GT family enzymes responsible for glycosylation on the carboxyl groups of triterpenoids remain unknown and should be investigated from the B. subtilis ATCC 6633 strain in the future. In addition to the GT families, some glycoside hydrolase (GH) families, such as the GH13 and GH70 families, have been reported to possess glycosyltransferase activity and accept small molecules, such as flavonoids, as sugar acceptors $[17,30]$. Thus, these GH enzymes of B. subtilis ATCC 6633 might also catalyze glycosylation on the carboxyl group of triterpenoids. Further studies to elucidate such catalytic enzymes will be undertaken in our laboratory.

Glycosylation is a common modification reaction in the biosynthesis of natural compounds. GT1 proteins are powerful enzymes in the glycosylation of natural compounds for in vivo or in vitro use. Glycosylation enhances the lipophilicity and water solubility of natural compounds. The development of a new biotransformation system of triterpenoid glycosylation would expand the search for new triterpenoids and the applications of the newly produced triterpenoids in medicine, cosmetics, and pharmacology use in the future. 


\section{Materials and Methods}

\subsection{Microorganism and Chemicals}

B. subtilis ATCC 6633 (BCRC 10447) was purchased from BCRC (Food Industry Research and Development Institute, Hsinchu, Taiwan). Antcin K was obtained by the procedure in our previous study [14]. GAA was bought from Baoji Herbest Bio-Tech (Xi-An, Shaanxi, China). UDP-glucose was purchased from Cayman Chemical (Ann Arbor, MI, USA). The other chemicals, materials needed for PCR, restriction enzymes, reagents and solvents used were of high quality, and were purchased from commercially available sources.

\subsection{Identification of Bacteria B. subtilis ATCC 6633 with Biotransformation Activity}

B. subtilis ATCC 6633 was cultivated in $20 \mathrm{~mL}$ of a modified glucose-nutrient (MGN) medium which contained $5 \mathrm{~g} / \mathrm{L}$ of peptone, yeast extract, $\mathrm{K}_{2} \mathrm{HPO}_{4}$, and $\mathrm{NaCl} ; 20 \mathrm{~g} / \mathrm{L}$ of glucose [14] and $20 \mathrm{mg} / \mathrm{L}$ of GAA. The cultivation was carried out at $180 \mathrm{rpm}$ and $28^{\circ} \mathrm{C}$ for $24 \mathrm{~h}$. After cultivation, $1 \mathrm{~mL}$ of the culture was then mixed with an equal volume of methanol and analyzed with UPLC.

\subsection{UPLC}

The UPLC system (Acquity UPLC H-Class, Waters, Milford, MA, USA) was equipped with an analytic C18 reversed-phase column (Kinetex ${ }^{\circledR} \mathrm{C} 18,1.7 \mu \mathrm{m}, 2.1$ i.d. $\times 100 \mathrm{~mm}$, Phenomenex Inc., Torrance, CA, USA). The operation conditions for UPLC of GAA and antcin K were from our previous study [14].

\subsection{Expression and Purification of GT1 of B. subtilis ATCC 6633}

The genomic DNA of B. subtilis ATCC 6633 was isolated using the commercial kit Geno Plus ${ }^{\mathrm{TM}}$ (Viogene, Taipei, Taiwan). BsUGT398 and BsUGT489 of B. subtilis ATCC 6633 were amplified from the genomic DNA with PCR with specific primer sets (Table S1). The amplified GT1 genes were subcloned into the pETDuet-1 $1^{\mathrm{TM}}$ vector through suitable restriction enzyme sites (Table S1) to obtain the expression vector pETDuet-UGT (Figure S2a). The expression vectors were transformed into E. coli BL21 (DE3) via electroporation to obtain recombinant E. coli.

Recombinant BsUGT398 and BsUGT489 were produced and purified from the recombinant E. coli. The experimental procedure was the same as our previous study [25].

\subsection{In Vitro Biotransformation Assay}

In vitro biotransformation was performed by purified GT1 protein. In $1 \mathrm{~mL}$ reaction mixture, $2 \mu \mathrm{g}$ of purified GT1 protein, $0.02 \mathrm{mg} / \mathrm{mL}$ of GAA or antcin $\mathrm{K}, 0.4 \mathrm{mM}$ of UDP-glucose, $10 \mathrm{mM}$ of $\mathrm{MgCl}_{2}$, and $50 \mathrm{mM}$ of Tris $\mathrm{pH} 8.0$ were added. The reaction was carried out at $40{ }^{\circ} \mathrm{C}$ for $30 \mathrm{~min}$. After the reaction, the mixture was stopped by adding an equal volume of methanol and analyzed with UPLC. For the optimal experiments, $2 \mu \mathrm{g}$ of the purified BsUGT398 or BsUGT489, $1 \mathrm{mg} / \mathrm{mL}$ of GAA, and $10 \mathrm{mM}$ of UDP-glucose were used. The $\mathrm{pH}$, temperature, or metal ion described above was replaced by the tested condition. For $\mathrm{pH}$ testing, phosphate buffer at $\mathrm{pH} 6.0$ and 7.0) and Tris buffer at pH 8.0 and 9.0 were used. For metal ion testing, $10 \mathrm{mM}$ of $\mathrm{MgCl}_{2}, \mathrm{CaCl}_{2}, \mathrm{CdSO}_{4}$ or $\mathrm{MnCl}_{2}$ was used. The relative activity was obtained by dividing the area of the product peak of the reaction in the UPLC profile by that of the reaction at Tris $\mathrm{pH} 8.0$ and $10 \mathrm{mM}$ of $\mathrm{MgCl}_{2}$.

\subsection{Scale-Up, Isolation, and Identification of the Biotransformation Product}

To purify the biotransformation metabolite, the reaction was scaled up to a $40 \mathrm{~mL}$ reaction mixture containing $20 \mu \mathrm{g}$ of purified BsUGT398 or BsUGT489, $1 \mathrm{mg} / \mathrm{mL}$ of GAA, $10 \mathrm{mM}$ of UDP-glucose, $10 \mathrm{mM}$ of $\mathrm{MgCl}_{2}$, and $50 \mathrm{mM}$ of Tris $\mathrm{pH}$ 8.0. After reaction at $30^{\circ} \mathrm{C}$ (BsUGT489) or $40{ }^{\circ} \mathrm{C}$ (BsUGT398) for $60 \mathrm{~min}, 40 \mathrm{~mL}$ of methanol was added to stop the reaction. The reaction mixture was filtrated 
through a $0.2 \mu \mathrm{m}$ nylon membrane, and applied into a preparative YoungLin HPLC system (YL9100, YL Instrument, Gyeonggi-do, South Korea). The system was equipped with a preparative C18 reversed-phase column (Inertsil, $10 \mu \mathrm{m}, 20.0$ i.d. $\times 250 \mathrm{~mm}$, ODS 3, GL Sciences, Eindhoven, The Netherlands). The operational conditions for the preparative HPLC were the same as those in UPLC. The elution corresponding to the peak of the metabolite in UPLC was collected, concentrated under vacuum, and then lyophilized. Finally, $15.1 \mathrm{mg}$ and $37.6 \mathrm{mg}$ of compound (1) were obtained from the BsUGT398 and BsUGT489 biotransformation, respectively, and the structure of the compound was confirmed with NMR and mass spectrometry analysis. The mass spectrometry analysis was performed on a Finnigan LCQ Duo mass spectrometer (ThermoQuest Corp., San Jose, CA, USA) with electrospray ionization (ESI). For the NMR experiments, purified samples quantities ranged from $4 \mathrm{mg}$ to $8 \mathrm{mg}$ and were dissolved in $400 \mu \mathrm{L}$ pyridine- $d_{5}$, and ${ }^{1} \mathrm{H}$ - and ${ }^{13} \mathrm{C}-\mathrm{NMR}$, and distortionless enhancement by polarization transfer (DEPT), heteronuclear single quantum coherence (HSQC), heteronuclear multiple bond connectivity (HMBC), correlation spectroscopy (COSY), and nuclear Overhauser effect spectroscopy (NOESY) spectra were recorded on a Bruker AV-700 NMR spectrometer (Bruker Corp., Billerica, MA, USA) at ambient temperature. Standard pulse sequences and parameters from the Bruker library were used for the NMR experiments, and all chemical shifts were reported in parts per million (ppm, $\delta)$.

\subsection{Phylogenetic Analysis of GTs from B. subtilis ATCC 6633}

The unrooted phylogenetic tree of these candidate GT protein sequences was constructed with the maximum likelihood method, using Molecular Evolutionary Genetics Analysis (MEGA X) software [24] with 500 bootstrap replications, the general reversible mitochondrial model [23], and partial deletion.

\section{Conclusions}

Two new BsUGTs from B. subtilis ATCC 6633 were identified as transferring a glucose moiety to GAA, producing a new triterpenoid glucoside, GAA-15-O- $\beta$-glucoside. More than 10,000 microbial GT genes have been deposited in the CAZy database. However, less than $1 \%$ of these GT genes have been functionally characterized. The two BsUGTs were newly identified with novel triterpenoid glycosylation activity. The two B. subtilis BsUGTs are the only identified GTs with glycosylation activity toward Ganoderma triterpenoids. Thus, the present study has developed a novel biotransformation system which might be applied to numerous Ganoderma triterpenoids to create many new Ganoderma triterpenoid glucosides in the future.

Supplementary Materials: Supplementary materials can be found at http:/ /www.mdpi.com/1422-0067/19/ 11/3469/s1. Table S1: Nucleotide sequences of the primers used for amplification of GT1 in the present study. Figure S1: UPLC of the $24 \mathrm{~h}$ fermentation broth using the B. subtilis ATCC 6633 strain without the addition of GAA. Figure S2: Expression of BsUGT398 and BsUGT489 from B. subtilis ATCC 6633 in E. coli. Figure S3. The ${ }^{1} \mathrm{H}-\mathrm{NMR}\left(700 \mathrm{MHz}\right.$, pyridine- $\left.d_{5}\right)$ spectrum of metabolite derived from GAA biotransformation by BsUGT398. Figure S4. The ${ }^{13} \mathrm{C}-\mathrm{NMR}\left(176 \mathrm{MHz}\right.$, pyridine- $\left.d_{5}\right)$ spectrum of metabolite derived from GAA biotransformation by BsUGT398. Figure S5. The DEPT-90 and DEPT-135 $(176 \mathrm{MHz}$, pyridine- $d 5)$ spectrum of metabolite derived from GAA biotransformation by BsUGT398. Figure S6. The HSQC (700 MHz, pyridine- $\left.d_{5}\right)$ spectrum of metabolite derived from GAA biotransformation by BsUGT398. Figure S7. The HMBC (700 MHz, pyridine- $\left.d_{5}\right)$ spectrum of metabolite derived from GAA biotransformation by BsUGT398. Figure S8. The ${ }^{1} \mathrm{H}-{ }^{1} \mathrm{H}$ COSY $(700 \mathrm{MHz}$, pyridine- $d_{5}$ ) spectrum of metabolite derived from GAA biotransformation by BsUGT398. Figure S9. The NOESY (700 MHz, pyridine- $d_{5}$ ) spectrum of metabolite derived from GAA biotransformation by BsUGT398. Figure S10. The ${ }^{1} \mathrm{H}-\mathrm{NMR}\left(700 \mathrm{MHz}\right.$, pyridine- $d_{5}$ ) spectrum of metabolite derived from GAA biotransformation by BsUGT489. Figure S11. The ${ }^{13} \mathrm{C}-\mathrm{NMR}\left(176 \mathrm{MHz}\right.$, pyridine- $\left.d_{5}\right)$ spectrum of metabolite derived from GAA biotransformation by BsUGT489. Figure S12. The DEPT-90 and DEPT-135 (176 MHz, pyridine- $d 5$ ) spectrum of metabolite derived from GAA biotransformation by BsUGT489. Figure S13. The HSQC (700 MHz, pyridine- $\left.d_{5}\right)$ spectrum of metabolite derived from GAA biotransformation by BsUGT489. Figure S14. The HMBC (700 MHz, pyridine- $\left.d_{5}\right)$ spectrum of metabolite derived from GAA biotransformation by BsUGT489. Figure S15. The ${ }^{1} \mathrm{H}-{ }^{1} \mathrm{H}$ COSY $(700 \mathrm{MHz}$, pyridine- $d_{5}$ ) spectrum of metabolite derived from GAA biotransformation by BsUGT489. Figure S16. The NOESY (700 MHz, pyridine- $d_{5}$ ) spectrum of metabolite derived from GAA biotransformation by BsUGT489. 
Author Contributions: Conceptualization, T.-S.C.; data curation, C.-M.C., T.-Y.W., K.-Y.W., J.-Y.W., and T.-S.C.; methodology, C.-M.C., T.-Y.W., K.-Y.W., J.-Y.W., and T.-S.C.; project administration, T.-S.C.; writing-original draft, C.-M.C., T.-Y.W., J.-Y.W., and T.-S.C.; writing—review and editing, C.-M.C., T.-Y.W., J.-Y.W., and T.-S.C.

Funding: This research was financially supported by grants from the National Scientific Council of Taiwan (Project No. MOST 107-2221-E-024-005-).

Conflicts of Interest: The authors declare no conflicts of interest.

\author{
Abbreviations \\ GAA Ganoderic acid A \\ GT Glycosyltransferase \\ GT1 Glycosyltransferase family 1 \\ UDP Uridine diphosphate \\ UGT Uridine diphosphate-dependent glycosyltransferases
}

\title{
References
}

1. Shah, S.A.A.; Tan, H.L.; Sultan, S.; Faridz, M.A.B.M.; Shah, M.A.B.M.; Nurfazilah, S.; Hussain, M. Microbial-catalyzed biotransformation of multifunctional triterpenoids derived from phytonutrients. Int. J. Mol. Sci. 2014, 15, 12027-12060. [CrossRef] [PubMed]

2. Sultana, N.; Saify, Z.S. Enzymatic biotransformation of terpenes as bioactive agents. J. Enzym. Inhib. Med. Chem. 2013, 28, 1113-1128. [CrossRef] [PubMed]

3. Muffler, K.; Leipold, D.; Scheller, M.C.; Haas, C.; Steingroewer, J.; Bley, T.; Neuhaus, H.E.; Mirata, M.A.; Schrader, J.; Ulber, R. Biotransformation of triterpenes. Process Biochem. 2011, 46, 1-15. [CrossRef]

4. Parra, A.; Rivas, F.; Garcia-Granados, A.; Martinez, A. Microbial transformation of triterpenoids. Mini-Rev. Org. Chem. 2009, 6, 307-320. [CrossRef]

5. Wu, J.W.; Zhao, W.; Zhong, J.J. Biotechnological production and application of ganoderic acids. Appl. Microbiol. Biotechnol. 2010, 87, 457-466.

6. Xia, Q.; Zhang, H.; Sun, X.; Zhao, H.; Wu, L.; Zhu, D.; Yang, G.; Shao, Y.; Zhang, X.; Mao, X.; et al. A comprehensive review of the structure elucidation and biological activity of triterpenoids from Ganoderma spp. Molecules 2014, 19, 17478-17535. [CrossRef] [PubMed]

7. Kubota, T.; Asaka, Y. Structures of ganoderic acid A and B, two new lanostane type bitter triterpenes from Ganoderma lucidum (FR.) Karst. Helv. Chim. Acta 1982, 65, 611-619. [CrossRef]

8. Jiang, J.; Grieb, B.; Thyagarajan, A.; Sliva, D. Ganoderic acids suppress growth and invasive behavior of breast cancer cells by modulating AP-1 and NF-kB signaling. Int. J. Mol. Med. 2008, 21, 577-584. [PubMed]

9. Yao, X.; Li, G.; Xu, H.; Lu, C. Inhibition of the JAK-STAT3 signaling pathway by ganoderic acid A enhances chemosensitivity of HepG2 cells to cisplatin. Planta Med. 2012, 78, 1740-1748. [CrossRef] [PubMed]

10. Wang, X.; Sun, D.; Tai, J.; Wang, L. Ganoderic acid A inhibits proliferation and invasion, and promotes apoptosis in human hepatocellular carcinoma cells. Mol. Med. Rep. 2017, 16, 3894-3900. [CrossRef] [PubMed]

11. Akihisa, T.; Nakamura, Y.; Tagata, M.; Tokuba, H.; Yasukawa, K.; Uchiyama, E.; Suzukli, T.; Kimura, Y. Anti-inflammatory and anti-tumor-promoting effects of triterpene acids and sterols from the fungus Ganoderma lucidum. Chem. Biod. 2007, 4, 224-231. [CrossRef] [PubMed]

12. Koyama, K.; Imaizumi, T.; Akiba, M.; Kinoshita, K.; Takahashi, K.; Suzuki, A.; Yano, S.; Horie, S.; Watanabe, K.; Naoi, Y. Antinociceptive components of Ganoderma lucidum. Planta Med. 1997, 63, 224-227. [CrossRef] [PubMed]

13. Zhu, M.; Chang, Q.; Wong, L.K.; Chong, F.S.; Li, R.C. Triterpene antioxidants from Ganoderma lucidum. Phytother. Res. 1999, 13, 529-531. [CrossRef]

14. Chang, T.S.; Chiang, C.M.; Siao, Y.Y.; Wu, J.Y. Sequential biotransformation of antcin K by Bacillus subtilis ATCC 6633. Catalysts 2018, 8, 349. [CrossRef]

15. Tiwari, P.; Sangwan, R.S.; Sangwan, NS. Plant secondary metabolism linked glycosyltransferases: An update on expanding knowledge and scopes. Biotechnol. Adv. 2016, 34, 716-739. [CrossRef] [PubMed]

16. Kim, B.G.; Yang, S.M.; Kim, S.Y.; Cha, M.N.; Ahn, J.H. Biosynthesis and production of glycosylated flavonoids in Escherichia coli: Current state and perspectives. Appl. Microbiol. Biotechnol. 2015, 99, 2979-2988. [CrossRef] [PubMed] 
17. Hofer, B. Recent developments in the enzymatic O-glycosylation of flavonoids. Appl. Microbiol. Biotechnol. 2016, 100, 4269-4281. [CrossRef] [PubMed]

18. CAZy Database. Available online: www.cazy.org (accessed on 10 October 2018).

19. Dai, L.; Li, J.; Yang, J.; Zhu, Y.; Men, Y.; Zeng, Y.; Cai, Y.; Dong, C.; Dai, Z.; Zhang, X.; et al. Use of a promiscuous glycosyltransferase from Bacillus subtilis 168 for the enzymatic synthesis of novel protopanaxtriol-type ginsenosides. J. Agric. Food Chem. 2017, 66, 943-949. [CrossRef] [PubMed]

20. Dai, L.; Li, J.; Yao, P.; Zhu, Y.; Men, Y.; Zeng, Y.; Yang, J.; Sun, Y. Exploiting the aglycon promiscuity of glycosyltransferase Bs-YjiC from Bacillus subtilis and its application in synthesis of glycosides. J. Biotechnol. 2017, 248, 69-76. [CrossRef] [PubMed]

21. Liang, H.; Hu, Z.; Zhang, T.; Gong, T.; Chen, J.; Zhu, P.; Li, Y.; Yang, J. Production of a bioactive unnatural ginsenoside by metabolically engineered yeasts based on a new UDP-glycosyltransferase from Bacillus subtilis. Metab. Eng. 2017, 44, 60-69. [CrossRef] [PubMed]

22. Wang, D.D.; Jin, Y.; Wang, C.; Kim, Y.J.; Perez, J.E.J.; Baek, N.I.; Mathiyalagan, R.; Markus, J.; Yang, D.C. Rare ginsenoside Ia synthesized from F1 by cloning and overexpression of the UDP-glycosyltransferase gene from Bacillus subtilis: Synthesis, characterization, and in vitro melanogenesis inhibition activity in BL6B16 cells. J. Ginseng Res. 2018, 42, 42-49. [CrossRef] [PubMed]

23. Adachi, J.; Hasegawa, M. Model of amino acid substitution in proteins encoded by mitochondrial DNA. J. Mol. Evol. 1996, 42, 459-468. [CrossRef] [PubMed]

24. Kumar, S.; Stecher, G.; Li, M.; Knyaz, C.; Tamura, K. MEGA X: Molecular evolutionary genetics analysis across computing platforms. Mol. Biol. Evol. 2018, 35, 1547-1549. [CrossRef] [PubMed]

25. Chiang, C.M.; Wang, T.Y.; Yang, S.Y.; Wu, J.Y.; Chang, T.S. Production of new isoflavone glucosides from glycosylation of 8-hydroxydaidzein by glycosyltransferase from Bacillus subtilis ATCC 6633. Catalysts 2018, 8, 349. [CrossRef]

26. Ünligil, U.; Rini, J.M. Glycosyltransferase structure and mechanism. Curr. Opin. Struct. Biol. 2000, 10, 510-517. [CrossRef]

27. Lariviere, L.; Gueguen-Chaignon, V.; Morera, S. Crystal structures of the T4 phage b-glucosyltransferase and the D100A mutant in complex with UDP-glucose: Glucose binding and identification of the catalytic base for a direct displacement mechanism. J. Mol. Biol. 2003, 330, 1077-1086. [CrossRef]

28. Chang, T.S.; Chiang, C.M.; Wang, T.Y.; Lee, C.H.; Lee, Y.W.; Wu, J.Y. New triterpenoid from novel triterpenoid 15-O-glycosylation on ganoderic acid A by intestinal bacteria of zebrafish. Molecules 2018, 23, 2345. [CrossRef] [PubMed]

29. Wang, W.W.; Xu, S.H.; Zhao, Y.Z.; Zhang, C.; Zhang, Y.Y.; Yu, B.Y.; Zhang, J. Microbial hydroxylation and glycosylation of pentacyclic triterpenes as inhibitors on tissue factor procoagulant activity. Bioorg. Med. Chem. Lett. 2017, 27, 1026-1030. [CrossRef] [PubMed]

30. Moulis, C.; Andre, I.; Remaud-Simeon, M. GH13 amylosucrases and GH70 branching sucrases, atypical enzymes in their respective families. Cell. Mol. Life Sci. 2016, 73, 2661-2679. [CrossRef] [PubMed] 